\title{
P04-6-2 Poster session
}

\section{Gene landscape and correlation between B cell infiltration and PD-L1 expression by analyses of TCGA lung adenocarcinoma patients}

\author{
Kuo-Hao Ho ${ }^{1,2}$, Peng-Hsu Chen ${ }^{1,2}$, Chwen-Ming Shih ${ }^{1,2}$, Ku-Chung Chen ${ }^{1,2}$ \\ ${ }^{I}$ Graduate Institute of Medical Sciences, College of Medicine, Taipei Medical University, Taiwan, ${ }^{2}$ Department of \\ Biochemistry and Molecular Cell Biology, School of Medicine, College of Medicine, Taipei Medical University, Taiwan
}

Background: Tumor infiltrating lymphocytes are related to positive clinical prognoses in numerous kinds of cancer. Programmed death ligand 1 (PD-L1), a mediator of the PD-1 receptor, exhibits an inhibitory role in cancer immune responses. Several studies identified that upregulation of PD-L1 impeded infiltrating $\mathrm{T}$ cell functions in lung adenocarcinoma (LUAD), a subtype of lung cancer. However, associations between PD-L1 levels and infiltrating B cells, another important immune regulator, remain unknown. Hence, we aimed to investigate the role of infiltrating $B$ cells in LUAD progression and its relation with PD-L1 expression.

Methods and results: The Cancer Genome Atlas (TCGA) LUAD dataset was utilized to explore associations among B cell infiltration, PD-L1, and LUAD patients' clinical outcomes. We found that high B cell infiltration was correlated with better prognoses, but PD-L1 might interfere with the survival advantage in patients with high B cell infiltration. In addition, the gene landscape with distinct PD-L1 levels of high infiltrating B cell populations was also comprehensively characterized. Using a gene set enrichment analysis, five upregulated signaling pathways were obtained: apoptosis, tumor necrosis factor (TNF) - $\alpha$ signaling via nuclear factor (NF)- $\kappa \mathrm{B}$, the apical surface, the interferon- $\alpha$ response, and KRAS signaling. Furthermore, four candidate genes and their related target drugs were provided by a drug enrichment analysis: interleukin- $2 \beta$ receptor (IL2RB), IL-2 $\gamma$ receptor (IL2RG), toll-like receptor 8 (TLR8), and TNF.

Conclusions: These findings suggest that tumor infiltrating $B$ cells could act as a clinical factor for considering anti-PD-L1 immunotherapy in LUAD. 